\title{
Prevalence of IGFBP3, NOS3 and TCF7L2 Polymorphisms and Their Association With Hypertension: A Population-Based Study With Brazilian Women of African Descent
}

\section{Abel Lira ( $\nabla$ abel.neto@arapiraca.ufal.br)}

UFAL: Universidade Federal de Alagoas https://orcid.org/0000-0001-7597-3761

\section{Nancy Vasconcelos}

UFAL: Universidade Federal de Alagoas

\section{Tamara Santos}

UFAL

Luisa Duarte

UFAL

\section{Monica Assunção}

UFAL

\section{Carolinne Marques}

UFAL: Universidade Federal de Alagoas

\section{Haroldo Ferreira}

UFAL: Universidade Federal de Alagoas

Research article

Keywords: Nitric Oxide Synthase, Hypertension, IGFBP3 Human Protein, Oxidative Stress, African Continental Ancestry Group

Posted Date: December 30th, 2020

DOl: https://doi.org/10.21203/rs.3.rs-136139/v1

License: (c) (i) This work is licensed under a Creative Commons Attribution 4.0 International License. Read Full License 


\section{Abstract}

Background: African ancestry seems to be a risk factor for hypertension; however, few genetic studies have addressed this issue. This study aimed to investigate the prevalence of polymorphisms NOS3; rs1799983, IGFBP3; rs11977526 and TCF7L2; rs7903146 in Brazilian women of African descent and their association with hypertension.

Methods: This is a cross-sectional study with a sample of 1021 women (19-59 years old) from the quilombola communities of Alagoas (Brazil). Demographic, socioeconomic, lifestyle, anthropometric, biochemical, and blood pressure data were collected. DNA was extracted from mucosa epithelial cells of the participants' cheek. Genotyping was performed by PCR allelic discrimination. Prevalence ratio (PR) was the measure of association, calculated by Poisson regression, with a hierarchical selection of variables.

Results: The prevalences of the less frequent genotypes were 26.5\% TT genotype of NOS3; rs 1799983, $16.7 \%$ AA genotype of IGFBP3; rs 11977526, and 18.3\% TT genotype of TCF7L2; rs7903146. For these conditions, the prevalence of hypertension and PR (adjusted) relatively to the ancestral genotype were, respectively: $52.0 \%$ vs $24.5 \%$ ( $P R=1.54 ; p<0.001), 62.0 \%$ vs $24.1 \%(P R=1.59 ; p<0.001)$, and $38.9 \%$ vs $27.9 \%(\mathrm{PR}=0.86 ; p=0.166)$. Associations with hypertension were statistically significant, except for the TCF7L2; rs7903146 polymorphism, after adjusted analysis.

Conclusions: Brazilian Afro-descendant women with the TT genotype for the NOS3 gene and the AA genotype for the IGFBP3 gene are more susceptible to hypertension. The understanding of underlying mechanisms involving the pathogenesis of hypertension can motivate research for the development of new therapeutic targets related to nitric oxide metabolism and the management of oxidative stress.

\section{Background}

Arterial hypertension characterized by important structural changes the vascular system in response to changes in hemodynamic conditions. It is intimately associated with other morbidities, such as dyslipidemia and diabetes mellitus, and is one of the most prevalent chronic non-communicable diseases in the world [1]. This disease is often associated with metabolic disorders, functional and/or structural changes in target organs. It can be aggravated by the presence of other risk factors, such as dyslipidemia, abdominal obesity, glucose intolerance, and diabetes mellitus [2].

Considered a disease of multifactorial etiology, hypertension is more prevalent among people of African descent. Therefore, it is important to understand the impact of the genetic component on the genesis of this pathology, with emphasis on polymorphisms [3]. Although there is a higher prevalence of hypertension in Afro-descendant populations in comparison with other ethnicities, studies involving the association of single nucleotide polymorphism (SNP) with this pathology have mostly been conducted with people of European ancestry, and few studies are dealing with populations of African origin $[4,5]$. 
Hypertension is a condition characterized by endothelial dysfunction, which is a phenomenon that, although discussed as being primary or secondary to hypertension, has a fundamental importance in its genesis and maintenance [6]. hypertension is also accompanied by structural changes in the vascular system in response to changes in hemodynamic conditions. The endothelial dysfunction of hypertension is mainly characterized by a non-relaxation of blood vessels caused by lower bioactivity of nitric oxide (NO) in the vascular wall, due to oxidative stress, causing an imbalance between the antioxidant and prooxidant systems, and leading to the prevalence of deleterious actions of reactive oxygen species on cells, tissues and organs $[7,8]$.

IGFBP3 is a protein with the function of regulating the bioavailability of IGF-1 [9]. In vitro experiments indicate that IGFBP3 regulates IGF-1 by reducing vascular resistance when stimulating the synthesis of nitric oxide in endothelial cells [10]. Therefore, IGFBP3 serum levels are closely related to the production of endothelial nitric oxide and, consequently, to oxidative stress and hypertension [11].

Sedentary lifestyle, visceral adiposity, and insulin resistance are important risk factors for both hypertension and diabetes mellitus (DM). However, although several studies have demonstrated a relationship between the TCF7L2 gene and DM, its relationship with the prevalence of arterial hypertension and oxidative stress has not been investigated $[12,13]$.

The SNPs in the NOS3 rs1799983, IGFBP3 rs11977526, and TCF7L2 rs7903146 genes can directly influence the protein expression in the respective genes, making them important biomarkers for the development of hypertension. However, no studies addressing this association in Afro-descendant populations were found $[14,15]$.

Given the above, this study aimed to verify the prevalence of SNPs in the NOS3 rs1799983, IGFBP3 rs11977526, and TCF7L2 rs7903146 genes, as well as to investigate the possible association of SNPs occurrence with arterial hypertension in Afro-descendant women, in quilombola communities in the state of Alagoas, northeastern Brazil.

\section{Methods}

This is a household cross-sectional population-based survey, whose data were collected between April 2017 and January 2018 from women aged 19-59 years, living in remaining quilombola communities, in the state of Alagoas, Brazil.

In the sample size calculation, hypertension was the variable of interest, whose prevalence in women of African descent was estimated at $35.8 \%$ [16]. Due to the lack of accurate demographic data categorized by sex, it was used the number of families $(6,465)$ estimated by the State Department of Planning, Management, and Heritage of Alagoas State (2015). Thus, it was considered that there would be a woman in each family, totaling 6,465 people. The calculations were performed using the StatCalc software (Epi Info, version 3.5.4). For a sampling error of 3.0\%, a $95 \%$ confidence interval, and adding $10 \%(852+85)$, to compensate for possible sample losses, 937 women would be needed. 
Using the systematic sampling strategy, $50 \%$ of the 68 quilombola communities of Alagoas were selected, which are distributed in 27 of the 102 municipalities of the State; the majority located between the 'Agreste' and 'Sertão' regions. In each residence, a woman aged 19-59 was eligible for the study. When there were two or more women, one of them was selected by lot. Being pregnant or in the postpartum period, as well as having drunk alcohol on the day of the interview were exclusion criteria.

The data were collected through interviews using structured questionnaires. During the interviews, blood pressure was measured and anthropometric data were obtained. Then, the women were referred to take biochemical tests in a predetermined place in each community (health service, school, community center, or any other place deemed more appropriate in the context of the respective community).

Hypertension was the dependent variable, defined by systolic blood pressure (SBP) $\geq 140 \mathrm{mmHg}$ and/or diastolic blood pressure (DBP) $\geq 90 \mathrm{mmHg}$ and/or when the participant reported regular use of antihypertensive drugs. The blood pressure was measured in duplicate, with the participant seated and after 15 minutes of rest, using Omron ${ }^{\circledR}$ digital devices (model HEM-7113). When there was a difference greater than $20 \mathrm{mmHg}$ between the two measurements, a third one was performed. To calculate the mean, for the analyses, the most discrepant value was disregarded [17].

The NOS3; rs1799983, IGFBP3; rs11977526 and TCF7L2; rs7903146 polymorphisms were the independent variables. For DNA extraction and polymorphisms testing, cell samples were collected from the women's oral mucosa. They were previously asked to rinse their mouth with $100 \mathrm{ml}$ of distilled water and the collection was performed by scraping the cheek mucosa with small sterile cytological brushes, making circular movements (approximately 30 times). Subsequently, the bristle part of the brushes was removed and placed into $2 \mathrm{ml}$ microtubes. The samples were stored in a refrigerator for subsequent DNA extraction using the salting-out method [18].

The gene encoding the endothelial nitric-oxide synthase NOS3, responsible for the production of NO, is located on chromosome 7 (gene locus 7q36). The NOS3; rs1799983 SNP is responsible for replacing a residue of glutamic acid with another of aspartic acid (Glu298Asp) [19]. The IGFBP3 gene is responsible for encoding the insulin-like growth factor binding protein and is located on chromosome 7 (7q12.3); rs11977526, SNP, an intron variant, is in a gene locus associated with IGFBP3 concentrations [20]. The TCF7L2 gene is responsible for encoding the TCF7L2 protein, which acts as a transcription factor. In humans, it is located on chromosome 10 (10q25.2). The $746 C>T$ (rs7903146) SNP is an intron variant [21].

The NOS3, rs1799983, IGFBP3, rs11977526, and TCF7L2, rs7903146 SNPs were chosen for this study after bibliographic research in a complete genome database (Genome-Wide Association Studies - GWAS) $[22,23]$. Genotyping was performed using the Step One Plus ${ }^{\text {TM }}$ Real-Time PCR System (Applied Biosystems, Foster City, CA, USA), based on a previously standardized protocol [18].

The following covariables were used to control possible confounding factors and characterize the sample: 
Demographic variables: age (19-30, 30.1-40, and 40.1-59 years).

Socioeconomic variables: waste disposal (public garbage collection and others); participation in the "Bolsa Família" Program, which is a social assistance intervention of the federal government based on income supplementation (yes or no); employment situation (formal, informal, unemployed, retired, pensioner); per capita family income ( $\geq 1$ minimum wage and $<1$ minimum wage); single register for social programs (yes or no); schooling level ( $\leq 4$ years, $>4$ years); self-reported skin color (caucasian, hispanic, african, asian, indigenous).

Regarding race/color, only two categories were considered in the statistical analysis: african / hispanic and others (caucasian, asian, indigenous). This categorization was necessary because of the small number of participants who declared themselves to be asian or indigenous $(n=9)$.

The food and nutrition security (FNS) or food and nutrition insecurity (FNI) was measured based on the Brazilian Food Insecurity Scale (EBIA), which classifies the home situation as security and mild, moderate, and severe insecurity. The validity of this instrument for quilombola communities was evidenced from the observation that families of such ethnicity were included in the sample used during the validation process $[24,25]$.

Variables related to health and lifestyle: Alcoholism (yes or no); smoking (yes or no); physical activity level (PAL) measured based on the results obtained by applying the International Physical Activity Questionnaire (IPAQ), short version 8, classifying women as sedentary or active, according to the obtained scores [26].

Anthropometric indicators: Body mass index $\left(\mathrm{BMl} ; \mathrm{kg} / \mathrm{m}^{2}\right)$ and waist circumference (WC) were used. To calculate the $\mathrm{BMI}$ of the participants, their weight and height were measured. For body mass measurement, portable digital scales (Seca ${ }^{\circledR}$, model 813), with a capacity of $200 \mathrm{~kg}$ and subdivisions of $100 \mathrm{~g}$ and calibrated weekly against standard weight, were used. Height was measured using a Seca ${ }^{\circledR}$ stadiometer 213 , equipped with a $205 \mathrm{~cm}$ inextensible tape measure subdivided in millimeters. The cutoff point proposed by the World Health Organization were used, obtaining the following categories: eutrophy (18.5-24.9 kg/m²), overweight $\left(25.0-29.9 \mathrm{~kg} / \mathrm{m}^{2}\right)$, and obesity $\left(\geq 30.0 \mathrm{~kg} / \mathrm{m}^{2}\right)$. Women with BMI 18.5 ( $n=18)$, which defines low body weight (thinness), were analyzed together with those classified as eutrophic. The WC was measured with the woman standing, using a non-extensible tape measure, which was positioned at the midpoint between the last rib and the iliac crest. A cut-off point $\geq 80 \mathrm{~cm}$ was used to identify high cardiovascular risk or metabolic complications associated with obesity [27].

Biochemical variables: Total cholesterol and fractions, triglycerides, and glycated hemoglobin (HbA1c) were determined without mandatory fasting. These analyses were performed using capillary blood obtained by puncturing the fingertip with disposable lancets. The lipid profile was determined in an Alere Cholestech LDX ${ }^{\circledR}$ System (Abbott, USA). Dyslipidemia was classified according to the cut-off points recommended by the Brazilian Guideline for Dyslipidemia and Atherosclerosis Prevention for samples 
obtained without previous fasting, as follow: cholesterol $\geq 190 \mathrm{mg} / \mathrm{dl}$, low-density lipoprotein (LDL) $\geq 160$ $\mathrm{mg} / \mathrm{dl}$, high density lipoprotein (HDL) of $50 \mathrm{mg} / \mathrm{dl}$, and triglycerides $\geq 175 \mathrm{mg} / \mathrm{dl}$ [28]. Diabetes mellitus was diagnosed based on $\mathrm{HbA} 1 \mathrm{c}$ level of $\geq 6.5 \%$ and/or the use of oral hypoglycemic agents. HbA1c was determined using a NycoCard Reader II ${ }^{\circledR}$ device (Abbott, USA) [29].

\section{Data processing and analysis}

Double independent data entry was performed using the Epi-Info ${ }^{\text {tm }} 3.5 .4$ software, identifying and correcting possible typing errors, after comparison. The obtained database was exported to the Stata/SE 12.1 software for Windows (StataCorp LP, College Station, TX, USA), through which all analyses were performed.The Hardy-Weinberg equilibrium (HWE) was analyzed using the $\chi^{2}$ test $(p>0.05)$. This procedure is necessary to certify that the study population obeys the principles of population genetics and, additionally, as a measure to control the accuracy obtained in the analysis of the genotypes [30].

To investigate differences in systolic blood pressure based on the genotypes of each polymorphism, distribution adherence to parametric assumptions was verified using the Kolmogorov-Smirnov test, complemented by graphical methods. Since this assumption was not clearly evidenced, it was decided to use both forms simultaneously. Thus, the means were subjected to analysis of variance (ANOVA), and the medians were tested using the Kruskal-Wallis test. Bonferroni and Dunnet post-hoc tests were used, respectively.

Multiple analysis was performed according to an adapted hierarchical theoretical model (NETO et al., 2019) (Figure 1), composed of four levels, as follows: (1) included demographic and socioeconomic variables, (2) variables related to lifestyle, (3) included anthropometric and biochemical indicators, and (4) associations with NOS3, rs1799983, IGFBP3, rs11977526, and TCF7L2, rs7903146, polymorphisms were analyzed. Level 2 was adjusted by the variables of level $1(p<0.05)$, level 3 was adjusted by the variables of levels 1 and $2(p<0.05)$, and level 4 was adjusted by all variables $(p<0.05)$ in the previous levels.

To identify an association between hypertension and the polymorphism genotypes, prevalence ratio (PR), and respective confidence interval $(95 \% \mathrm{Cl})$ were used, which were calculated using Poisson regression with robust variance. All covariables associated with hypertension $(p<0.2)$ in the crude analysis were eligible for hierarchical analysis. In each of the four levels of analysis, there was a successive exclusion of non-significant variables (backward stepwise elimination), remaining only those with $p<0.05$. From this definition, all these variables remained in the final adjusted model, even though they have lost statistical significance in the levels following their original level, in which they were inserted as control variables.

Two models were developed to verify the association between the oxidative stress model and the polymorphisms with a value of $p<0.05$ in the hierarchical analysis: (1) dominant, composed of ancestral genotypes vs heterozygous genotypes + less frequent genotypes, and (2) recessive model, composed of less frequent genotypes vs ancestral genotypes + heterozygous genotypes [31]. 


\section{Results}

A total of 1,091 women were eligible for this research. Those who had neither anthropometric $(n=8$; $0.73 \%)$ nor blood pressure $(n=4 ; 0.36 \%)$ data and those whose DNA was not extracted $(n=58 ; 5.31 \%)$ were excluded. Therefore, the analyzed final sample was composed of 1021 women ( $37.9 \pm 10.9$ years old), most of them self-declared as african / hispanic (91.1\%), with per capita family income below one minimum wage $(66.0 \%)$ and beneficiaries of the "Bolsa Família" Program (74.1\%). Half of them had up to four years of schooling (50.1\%) and $73.7 \%$ belonged to families in a situation of food insecurity.

Alcoholism and smoking were reported by $5.4 \%$ and $17.1 \%$ of women, respectively. Regarding physical activity, $61.2 \%$ of the women were classified as sedentary. The prevalence of hypertension among them was $31.4 \%$, and $26.6 \%$ were affected by diabetes. Changes corresponding to hypertriglyceridemia and low HDL were recorded in $32.3 \%$ and $40.3 \%$ of women, respectively. High total and LDL cholesterol were recorded in $44.4 \%$ and $25.9 \%$ of the participants, respectively. The sample characterization according to socioeconomic, demographic, lifestyle, anthropometric, biochemical, and genetic variables are shown in Table 1. 
Table 1

Distribution of systemic arterial hypertension the according to socioeconomic, demographic, lifestyle, anthropometric, and biochemical variables in women of African descent in the state of Alagoas $(n=1021)$.

\begin{tabular}{|c|c|c|c|}
\hline Variables & No. (\%) & Hypertension (\%) & *P-value \\
\hline Age group (years) & & - & - \\
\hline $19-30$ & $293(28.7)$ & 6.5 & - \\
\hline $30,1-40$ & $325(31.8)$ & 25.5 & $<0.001$ \\
\hline $40,1-59$ & 403(39.5) & 54.3 & $<0.001$ \\
\hline Public Garbage Collection & & - & - \\
\hline Yes & $498(48.9)$ & 29.6 & - \\
\hline No & $521(51.1)$ & 33.3 & 0.194 \\
\hline “Bolsa Família” Program & & - & - \\
\hline Yes & $757(74.1)$ & 27.2 & - \\
\hline No & $264(25.9)$ & 43.6 & $<0.001$ \\
\hline Unemployed & & - & - \\
\hline No & $551(54.3)$ & 34.6 & 0.017 \\
\hline Yes & $463(45.7)$ & 27.7 & - \\
\hline Per capita income & & - & - \\
\hline$\geq$ Minimum wage & $276(34.0)$ & 31.5 & - \\
\hline$<$ Minimum wage & $536(66.0)$ & 33.3 & 0.602 \\
\hline Single register for social programs & & - & - \\
\hline Yes & $801(78.4)$ & 28.3 & - \\
\hline No & $220(21.6)$ & 42.7 & $<0.001$ \\
\hline Schooling level & & - & - \\
\hline$>4$ anos & 509 (49.9) & 18.9 & - \\
\hline$\leq 4$ anos & $511(50.1)$ & 43.8 & $<0.001$ \\
\hline Skin color (self-reported) & & - & - \\
\hline Others & $91(8.9)$ & 28.6 & - \\
\hline African/Hispanic & $928(91.1)$ & 31.8 & 0.528 \\
\hline Food Insecurity & & - & - \\
\hline
\end{tabular}




\begin{tabular}{|c|c|c|c|}
\hline Variables & No. (\%) & Hypertension (\%) & *P-value \\
\hline No $(0)$ & $266(26.3)$ & 32.1 & - \\
\hline Yes $(\geq 1)$ & $746(73.7)$ & 31.2 & 0.657 \\
\hline Alcoholism & & - & - \\
\hline No $(0)$ & $961(94.6)$ & 31.4 & - \\
\hline Yes $(\geq 1)$ & $55(5.4)$ & 32.7 & 0.840 \\
\hline Smoking (last three months) & & - & - \\
\hline No $(0)$ & $842(82.9)$ & 29.5 & - \\
\hline Yes $(\geq 1)$ & $174(17.1)$ & 41.4 & $<0.002$ \\
\hline Physical activity level & & - & - \\
\hline Active & $394(38.8)$ & 31.5 & - \\
\hline Sedentary & $622(61.2)$ & 31.9 & 0.83 \\
\hline BMI classification $\left(\mathrm{kg} / \mathrm{m}^{2}\right)$ & & - & - \\
\hline Eutrophy $(18,5 a<25)$ & $315(31.3)$ & 19.6 & - \\
\hline Overweight $(\geq 25 a<30)$ & $377(37.5)$ & 32.6 & $<0.001$ \\
\hline Obesity $(\geq 30)$ & $313(31.1)$ & 42.5 & $<0.001$ \\
\hline Waist circumference & & - & - \\
\hline Normal $(<0.80)$ & $325(32.5)$ & 17.2 & - \\
\hline Increased $(\geq 0.80)$ & $676(67.5)$ & 38.8 & $<0.001$ \\
\hline Diabetes Mellitus & & - & - \\
\hline No $(0)$ & $749(73.4)$ & 26.0 & - \\
\hline Yes $(\geq 1)$ & $272(26.6)$ & 46.3 & $<0.001$ \\
\hline Triglycerides (mg/dL) & & - & - \\
\hline Normal $(<175)$ & $691(67.8)$ & 24.8 & - \\
\hline $\operatorname{High}(\geq 175)$ & $329(32.3)$ & 45.6 & $<0.001$ \\
\hline Total cholesterol (mg/dL) & & - & - \\
\hline Normal $(<190)$ & $568(55.6)$ & 23.9 & - \\
\hline $\operatorname{High}(\geq 190)$ & $453(44.4)$ & 40.8 & $<0.001$ \\
\hline *LDL-C (mg/dL) & & - & - \\
\hline
\end{tabular}




\begin{tabular}{|llll|}
\hline Variables & No. (\%) & Hypertension (\%) & *P-value \\
\hline Normal $(<130)$ & $703(74.2)$ & 29.5 & - \\
\hline High $(\geq 130)$ & $245(25.9)$ & 38.8 & $<0.007$ \\
*HDL-C $(\mathrm{mg} / \mathrm{dL})$ & & - & - \\
\hline Normal $(\geq 40)$ & $609(59.7)$ & 26.6 & - \\
\hline Low $(<40)$ & $411(40.3)$ & 38.4 & $<0.001$ \\
\hline
\end{tabular}

LDL: low-density lipoprotein; HDL: high density lipoprotein; hypertension: systemic arterial hypertension

P-value determined by the chi-square test

The prevalences of genotypes for NOS3; rs 1799983 SNP were GG $=47.9 \%, G T=25.6 \%$ and TT $=26.5 \%$, which was the least frequent genotype. The prevalences for TCF7L2; rs7903146 SNP was CC $=51.6 \%$, CT $=29.8 \%$ and $\mathrm{TT}=18.6 \%$. The IGFBP3; rs 11977526 SNP had the following prevalences: $\mathrm{GG}=39.5 \%, \mathrm{GA}=$ $43.8 \%$ and $A A=16.7 \%$. There was a statistically significant difference between blood pressure levels and the NOS3, rs1799983 and IGFBP3 rs11977526, SNPs, with emphasis on the genotypes: TT NOS3; rs1799983, SNP and AA IGFBP3; rs11977526 SNP.

The less frequent genotypes of the NOS3, rs1799983, TCF7L2 rs7903146, and IGFBP3 rs11977526, SNPs were associated with a higher prevalence of hypertension in comparison with the ancestral and heterozygous genotypes. The distribution of polymorphisms in accordance with the Hardy-Weinberg equilibrium (Table 2). 
Table 2

- Prevalence of arterial hypertension (\%) and systolic blood pressure (mean \pm SD; median and interquartile range), according to the genotypic frequencies for NOS3 rs1799983, TCF7L2 rs7903146, and IGFBP3 rs11977526 genes. Brazilian women descended from African descent, 2018.

\begin{tabular}{|c|c|c|c|c|}
\hline \multirow[t]{2}{*}{ Genotype } & \multirow{2}{*}{$\begin{array}{l}\text { Genotype frequency } \\
\text { (\%) }\end{array}$} & \multirow{2}{*}{$\begin{array}{l}\text { Hypertension } \\
\text { (\%) }\end{array}$} & \multicolumn{2}{|c|}{ Systolic blood pressure ${ }^{\bigotimes}$} \\
\hline & & & Mean \pm SD & $\begin{array}{l}\text { Median (P25 - } \\
\text { P75) }\end{array}$ \\
\hline \multicolumn{5}{|c|}{$\begin{array}{l}\text { NOS3 } \\
\text { rs1799983a }\end{array}$} \\
\hline GG & 489 (47.9) & 24.5 & $123.8 \pm 17.9$ & $\begin{array}{l}120.5(112.5- \\
131.0)\end{array}$ \\
\hline GT & $261(25.6)$ & 23.0 & $124.7 \pm 18.8$ & $\begin{array}{l}121.0(112.5- \\
132.0)\end{array}$ \\
\hline \multirow[t]{2}{*}{ TT } & $271(26.5)$ & 52.0 & $132.2 \pm 21.9 *$ & $\begin{array}{l}129.0(114.5- \\
147.5)^{\star \star}\end{array}$ \\
\hline & & & $\begin{array}{l}\mathrm{p}<0.001 \\
\text { (Anova) }\end{array}$ & $p=0.0001(K-W)$ \\
\hline \multicolumn{5}{|c|}{$\begin{array}{l}\text { TCF7L2 } \\
\text { rs7903146 }\end{array}$} \\
\hline $\mathrm{CC}$ & $527(51.6)$ & 27.9 & $125.44 \pm 19.21$ & $\begin{array}{l}122.0(113.0- \\
133.0)\end{array}$ \\
\hline CT & $304(29.8)$ & 32.9 & $125.86 \pm 19.13$ & $\begin{array}{l}121.0(113.5- \\
134.8)\end{array}$ \\
\hline \multirow[t]{2}{*}{ TT } & $190(18.6)$ & 38.9 & $129.13 \pm 21.15$ & $\begin{array}{l}124.5(113.5- \\
140.0)\end{array}$ \\
\hline & & & $\begin{array}{l}p=0.0775 \\
\text { (Anova) }\end{array}$ & $p=0.1118(K-W)$ \\
\hline \multicolumn{5}{|c|}{$\begin{array}{l}\text { IGFBP3 } \\
\text { rs11977526c }\end{array}$} \\
\hline GG & $403(39.5)$ & 24.1 & $123.49 \pm 18.04$ & $\begin{array}{l}120.0(111.5- \\
129.5)\end{array}$ \\
\hline AG & $447(43.8)$ & 26.4 & $125.49 \pm 19.04$ & $\begin{array}{l}121.0(112.5- \\
134.0)\end{array}$ \\
\hline \multirow[t]{2}{*}{ AA } & $171(16.7)$ & 62.0 & $134.78 \pm 22.13^{\star}$ & $\begin{array}{l}131.0(118.5- \\
147.5)^{\star \star}\end{array}$ \\
\hline & & & $\begin{array}{l}\mathrm{p}<0.001 \\
\text { (Anova) }\end{array}$ & $p=0.0001(K-W)$ \\
\hline
\end{tabular}

a, b, c Hardy Weinberg Equilibrium: 
NOS3, rs1799983: chi $^{2}=220.1159, p=0.606$

TCF7L2, rs7903146: chi² $^{2}=112.3333, p=0.665$

IGFBP3; rs11977526: $\mathrm{chi}^{2}=6.009 p=0.613$

${ }^{\sharp}$ One-sample Kolmogorov-Smirnov test against normal theoretical distribution: $p<0.001$ (systolic blood pressure distribution differs significantly from Gaussian distribution).

* Differs significantly from the others $(p<0.01$ according to the Bonferroni test).

$\mathrm{K}-\mathrm{W}=$ Kruskal-Wallis test .

** Differs significantly from the others ( $p<0.01$ according to the Dunnet test with Bonferroni correction). Results similar to those recorded for distribution of prevalence were found when analyzing the measures of central tendency (mean and median) related to systolic blood pressure (Table 2). The values found for genotypes of NOS3, rs1799983 and IGFBP3 rs 11977526 were significantly higher than those obtained for the other genes, both in the ANOVA, in the Kruskal-Wallis nonparametric test. For the TCF7L2, rs7903146 SNP, PAS levels were considered statistically similar ( $p>0.05$ in both analyses).

For the NOS3, rs1799983 SNP, the prevalences of hypertension among women with GG, GT and TT genotypes were $24.5 \%, 23.0 \%$ and $52.0 \%$, respectively, with a statistically significant difference between TT and GG (PR $=1.54 ; 95 \% \mathrm{Cl}=1.27-1.86 ; p<0.001)$. Regarding IGFBP3; rs11977526 SNP, the prevalences of hypertension among women with GG, GA and AA genotypes were $24.1 \%, 26.4 \%$ and $62.0 \%$, respectively, with a statistically significant difference between $A A$ and $G G(P R=1.59 ; 95 \% \mathrm{Cl}=$ 1.27-1.98; $p$ <0.001). For TCF7L2; rs7903146 SNP, hypertension among women with CC, CT and TT genotypes had prevalences of $27.9 \%, 32.9 \%$ and $38.9 \%$, respectively, with no statistical difference between TT and CC $(\mathrm{PR}=0.86 ; 95 \% \mathrm{Cl}=0.69-1.06 ; p=0.166)$ (Table 3$)$. 
Table 3

- Prevalence ratios (PR) and respective $95 \%$ confidence intervals $(95 \% \mathrm{Cl})$ obtained by multivariable Poisson regression, according to the hierarchical theoretical model for determining arterial hypertension.

\begin{tabular}{|c|c|c|c|c|c|c|c|}
\hline Variables & $\begin{array}{l}\text { Level } 1 \\
\text { PR (95\% } \\
\text { Cl) }\end{array}$ & $\begin{array}{l}P \\
\text { value }\end{array}$ & $\begin{array}{l}\text { Level } 2 \\
\text { *PR } \\
(95 \% \mathrm{Cl})\end{array}$ & $\begin{array}{l}P \\
\text { value }\end{array}$ & $\begin{array}{l}\text { Level } 3 \\
\text { *PR } \\
(95 \% \\
\text { Cl) }\end{array}$ & $\begin{array}{l}\mathrm{P} \\
\text { value }\end{array}$ & $\begin{array}{l}\text { Level } \\
\mathbf{4} \\
\text { PR } \\
\text { (95\% } \\
\text { Cl) }\end{array}$ \\
\hline
\end{tabular}

\section{Level 1}

\begin{tabular}{|c|c|c|c|c|c|c|c|c|}
\hline $\begin{array}{l}\text { Age group: } 30.1- \\
40\end{array}$ & $\begin{array}{l}3.95 \\
(2.46- \\
6.34)\end{array}$ & $<.001$ & $\begin{array}{l}3.95 \\
(2.46- \\
6.34)\end{array}$ & $<.001$ & $\begin{array}{l}3.54 \\
(2.20- \\
5.69)\end{array}$ & $\begin{array}{l}< \\
0.01\end{array}$ & $\begin{array}{l}3.36 \\
(2.10- \\
5.36)\end{array}$ & $\begin{array}{l}<.01 \\
0.01\end{array}$ \\
\hline $\begin{array}{l}\text { Age group: } 40.1- \\
50\end{array}$ & $\begin{array}{l}8.06 \\
(5.16- \\
12.60)\end{array}$ & $<.001$ & $\begin{array}{l}8.06 \\
(5.16- \\
12.60)\end{array}$ & $<.001$ & $\begin{array}{l}7.33 \\
(4.69- \\
11.48)\end{array}$ & $\begin{array}{l}< \\
0.01\end{array}$ & $\begin{array}{l}6.78 \\
(4.35- \\
10.59)\end{array}$ & $\begin{array}{l}< \\
0.01\end{array}$ \\
\hline $\begin{array}{l}\text { Insertion in the } \\
\text { formal labor } \\
\text { market }\end{array}$ & $\begin{array}{l}1.18 \\
(0.99- \\
1.41)\end{array}$ & 0.050 & $\begin{array}{l}1.18 \\
(0.99- \\
1.41)\end{array}$ & 0.051 & $\begin{array}{l}1.17 \\
(0.99- \\
1.40)\end{array}$ & 0.06 & * & * \\
\hline $\begin{array}{l}\text { Schooling level: } \leq \\
4 \text { years }\end{array}$ & $\begin{array}{l}1.21( \\
0.99- \\
1.48)\end{array}$ & 0.060 & $\begin{array}{l}1.21( \\
0.99- \\
1.48)\end{array}$ & 0.056 & * & * & * & * \\
\hline $\begin{array}{l}\text { "Bolsa Família" } \\
\text { Program: yes }\end{array}$ & $\begin{array}{l}1.20 \\
(1.01- \\
1.41)\end{array}$ & 0.037 & $\begin{array}{l}1.20 \\
(1.01- \\
1.41)\end{array}$ & 0.037 & $\begin{array}{l}1.15 \\
(0.98- \\
1.36)\end{array}$ & 0.094 & $\begin{array}{l}1.12 \\
(0.96- \\
1.31)\end{array}$ & 0.156 \\
\hline $\begin{array}{l}\text { Single register for } \\
\text { social programs: } \\
\text { yes }\end{array}$ & $\begin{array}{l}1.06 \\
(0.78- \\
1.44)\end{array}$ & 0.680 & * & * & * & * & * & * \\
\hline
\end{tabular}

\section{Level 2}

Smoking

$\begin{array}{ll}0.99 & 0.48 \text { * } \\ (0.77- & \\ 1.13) & \end{array}$

\section{Level 3}

\begin{tabular}{|c|c|c|c|c|c|c|c|c|}
\hline $\begin{array}{l}\text { BMI Overweight }(\geq \\
25-<30)\end{array}$ & & & & & $\begin{array}{l}1.26 \\
(0.98- \\
1.61)\end{array}$ & 0.067 & $\begin{array}{l}1.29 \\
(1.02- \\
1.64)\end{array}$ & 0.033 \\
\hline BMI Obesity $(\geq 30)$ & - & - & - & - & $\begin{array}{l}1.63 \\
(1.29- \\
2.06)\end{array}$ & $<.001$ & $\begin{array}{l}1.63 \\
(1.29- \\
2.06)\end{array}$ & $\begin{array}{l}< \\
0.001\end{array}$ \\
\hline $\begin{array}{l}\text { Waist } \\
\text { circumference } \geq \\
80 \mathrm{~cm}\end{array}$ & - & - & - & - & $\begin{array}{l}1.02 \\
(0.72- \\
1.43)\end{array}$ & 0.091 & & * \\
\hline
\end{tabular}




\begin{tabular}{|c|c|c|c|c|c|c|c|c|}
\hline Variables & $\begin{array}{l}\text { Level } 1 \\
\text { PR (95\% } \\
\mathrm{Cl})\end{array}$ & $\begin{array}{l}P \\
\text { value }\end{array}$ & $\begin{array}{l}\text { Level } 2 \\
\text { *PR } \\
(95 \% \mathrm{Cl})\end{array}$ & $\begin{array}{l}\mathrm{P} \\
\text { value }\end{array}$ & $\begin{array}{l}\text { Level } 3 \\
\text { *PR } \\
(95 \% \\
\mathrm{Cl})\end{array}$ & $\begin{array}{l}\mathrm{P} \\
\text { value }\end{array}$ & $\begin{array}{l}\text { Level } \\
4 \\
\text { PR } \\
(95 \% \\
\text { Cl) }\end{array}$ & $\begin{array}{l}\mathrm{P} \text { - } \\
\text { value }\end{array}$ \\
\hline $\begin{array}{l}\text { Triglycerides } \geq \\
175 \mathrm{mg} / \mathrm{dL}\end{array}$ & - & - & - & - & $\begin{array}{l}1.05 \\
(0.88- \\
1.26)\end{array}$ & 0.551 & * & * \\
\hline $\begin{array}{l}\text { Total cholesterol } \geq \\
190 \mathrm{mg} / \mathrm{dL}\end{array}$ & - & - & - & - & $\begin{array}{l}1.20 \\
(0.96- \\
1.49)\end{array}$ & 0.101 & * & * \\
\hline $\begin{array}{l}* L D L-C(\mathrm{mg} / \mathrm{dL}) \geq \\
160 \mathrm{mg} / \mathrm{dL}\end{array}$ & - & - & - & - & $\begin{array}{l}0.90( \\
0.73- \\
1.12)\end{array}$ & 0.384 & * & * \\
\hline $\begin{array}{l}{ }^{*} H D L-C(m g / d L)> \\
50 \mathrm{mg} / \mathrm{dL}\end{array}$ & - & - & - & - & $\begin{array}{l}1.27 \\
(1.08- \\
1.50)\end{array}$ & 0.003 & $\begin{array}{l}1.24 \\
(1.06- \\
1.45)\end{array}$ & 0.007 \\
\hline Diabetes mellitus & - & - & - & - & $\begin{array}{l}1.28 \\
(1.09- \\
1.51)\end{array}$ & 0.003 & $\begin{array}{l}1.16 \\
(0.99- \\
1.37)\end{array}$ & 0.060 \\
\hline \multicolumn{9}{|l|}{ Level 4} \\
\hline \multicolumn{9}{|l|}{ TCF7L2 } \\
\hline $\mathrm{CC}$ & - & - & - & - & - & - & 1 & - \\
\hline CT & - & - & - & - & - & - & $\begin{array}{l}1.04 \\
(0.78- \\
1.19)\end{array}$ & 0.623 \\
\hline TT & - & - & - & - & - & - & $\begin{array}{l}0.86 \\
(0.69- \\
1.06)\end{array}$ & 0.166 \\
\hline \multicolumn{9}{|l|}{ NOS3 } \\
\hline GG & - & - & - & - & - & - & 1 & - \\
\hline GT & - & - & - & - & - & - & $\begin{array}{l}0.95 \\
(0.74- \\
1.23)\end{array}$ & 0.755 \\
\hline TT & - & - & - & - & - & - & $\begin{array}{l}1.54( \\
1.27- \\
1.86)\end{array}$ & $\begin{array}{l}<.001 \\
0.001\end{array}$ \\
\hline \multicolumn{9}{|l|}{ IGFBP3 } \\
\hline GG & - & - & - & - & - & - & 1 & - \\
\hline
\end{tabular}




\begin{tabular}{|c|c|c|c|c|c|c|c|c|}
\hline Variables & $\begin{array}{l}\text { Level } 1 \\
\text { PR (95\% } \\
\text { Cl) }\end{array}$ & $\begin{array}{l}P \\
\text { value }\end{array}$ & $\begin{array}{l}\text { Level } 2 \\
\text { *PR } \\
(95 \% \mathrm{Cl})\end{array}$ & $\begin{array}{l}P \\
\text { value }\end{array}$ & $\begin{array}{l}\text { Level } 3 \\
\text { *PR } \\
\text { (95\% } \\
\text { Cl) }\end{array}$ & $\begin{array}{l}\mathrm{P} \\
\text { value }\end{array}$ & $\begin{array}{l}\text { Level } \\
4 \\
\text { PR } \\
(95 \% \\
\text { Cl) }\end{array}$ & $\begin{array}{l}\text { P- } \\
\text { value }\end{array}$ \\
\hline AG & - & - & - & - & - & - & $\begin{array}{l}0.96( \\
0.78- \\
1.19)\end{array}$ & 0.751 \\
\hline AA & - & - & - & - & - & - & $\begin{array}{l}1.59( \\
1.27- \\
1.98)\end{array}$ & $\begin{array}{l}< \\
0.001\end{array}$ \\
\hline
\end{tabular}

LDL: Iow-density lipoprotein; HDL: high density lipoprotein; Prevalence ratios and respective 95\% confidence intervals $(95 \% \mathrm{Cl})$

After the adjusted analysis, older age group, family beneficiary of the "Bolsa Família" Program, obesity $\left(B M I \geq 30 \mathrm{~kg} / \mathrm{m}^{2}\right)$, low HDL-C level, and diabetes mellitus were significantly associated with hypertension.

The dominant and recessive model analysis was performed using the following risk factors: age group (30.1-40.0), age group (40.1-59.0), "Bolsa Família" Program, overweight (BMI $\geq 25)$, HDL-C, and glycated hemoglobin. In the dominant model of oxidative stress (NOS3: GG + IGFBP3: GG vs NOS3 GT + TT; IGFBP3: AG + AA), women had a prevalence of 34.7\% hypertension ( $\mathrm{PR}=1.42 ; 95 \% \mathrm{Cl}: 1.12-1.79 ; p=$ 0.003). However, in the recessive model of oxidative stress (NOS3: TT + IGFBP3: AA vs NOS3: GG + GT IGFBP3: $\mathrm{GG}+\mathrm{AG})$, they had a prevalence of $77.0 \%$ hypertension ( $\mathrm{PR}=2.07 ; 95 \% \mathrm{Cl}: 1.78-2.42 p<0.001)$. There was a statistical significance even after adjusting for all risk factors, in the dominant and recessive models, referring to the NOS3 and IGFBP3 genes (Table 4).

Table 4

- Dominant and recessive models for determining systemic arterial hypertension in Brazilian women of African descent.

\begin{tabular}{|llll|}
\hline Models & Hypertension (\%) & *PR $(95 \% \mathrm{Cl})$ & P-value \\
\hline Dominant & & & \\
\hline (NOS3 GG $)+($ IGFBP3 GG) & 21.3 & 1 & - \\
\hline (NOS3 TT + GT $+($ IGFBP3 AG + AA) & 34.7 & $1.42(1.12-1.79)$ & 0.003 \\
\hline Recessive & & & - \\
\hline (NOS3 GG + GT $+($ IGFBP3 GG + AG) & 26.5 & 1 & $<0.001$ \\
\hline (NOS3 TT $)+($ IGFBP3 AA) & 77.0 & $2.07(1.78-2.42)$ & $<$ \\
\hline
\end{tabular}

Prevalence ratios (PR) and respective $95 \%$ confidence intervals $(95 \% \mathrm{Cl})$ adjusted by Poisson regression using the risk factors: age group (30.1-40.0), age group $(40.1-59,0)$, income supplementation program, overweight (BMI $\geq 25-<30)$, obesity (BMI $\geq 30), \mathrm{HDL}-\mathrm{C}$ and diabetes mellitus; * hypertension: systemic arterial hypertension 


\section{Discussion}

This study provides evidence that, in Afro-descendant women from northeastern Brazil, the NOS3, rs1799983 and IGFBP3, rs11977526, SNPs gene are associated with higher blood pressure levels and, consequently, with a higher prevalence of arterial hypertension. This same relationship had already been observed for the TCF7L2 rs7903146, SNP only in the crude analysis because after adjusted analysis there was no significance.

A study in an African population investigated whether biomarkers of endothelial function were related to the bioavailability of IGF-1 (IGF-1, IGFBP3, or IGF-1/IGFBP3M ratio) and showed that the bioavailable IGF1, measured by the IGF1/IGFBP3 ratio, is beneficially associated with CAV-1, which is a biomarker of endothelial activation [32]. Also, bioavailable IGF-1 tended to be inversely associated with ICAM-1, another marker of endothelial activation, thereby increasing the expression of CAV-1 and ICAM-1 [11, 33].

Previous studies indicated that some SNPs in the IGFBP3 and NOS3 genes are associated with decreased serum levels of these proteins [34-36]. Meta-analysis data from 11,700 pregnant women with gestational-induced hypertension, in African and Latin American populations, showed a relationship between G894T polymorphism and susceptibility to this type of hypertension in the dominant model [37]. Also, several studies showed a strong association between $894 \mathrm{G}>T$ polymorphism of NOS3 gene and hypertension $[18,38,39]$.

Research on SNPs of the IGFBP3 rs11977526 gene and hypertension indicated an association of these factors, as found in a study involving East African people, was associated with the risk of such disease [40] [41].

In this study, there was a higher predisposition for hypertension in the presence of TT and AA genotypes for the NOS3 and IGFBP3 genes, respectively. In the case of heterozygous Afro-descendant women (GT of the NOS3 gene and AG of the IGFBP3 gene), no statistically significant difference was found between the prevalence of hypertension. However, when analyzing the dominant and recessive models for oxidative stress, even after adjusting the risk factors, a significance was found for NOS3 and IGFBP3 models. Therefore, these data show that the presence of the TT genotype of the NOS3 gene and the AA genotype of the IGFBP3 gene constitutes an important risk factor for arterial hypertension.

The SNP rs1799983, variant of the NOS3 gene causes a change in which the amino acid Asp is replaced by Glu at position 298. This substitution is associated with a decrease in protein stability $[15,42]$. The AA genotype of the IGFBP3 gene has been shown to regulate protein expression through miRNAs by destabilizing the mRNA, which is associated with a decrease in IGFBP3 serum levels [20,41, 43].

In our analysis, the dominant and recessive showed a strong association of T and A alleles of the NOS3 and IGFBP3 genes, respectively, with hypertension. CAV-1 is the main link between the NOS3 gene and IGFBP3 because it physically interacts with these gene regions, making possible a co-expression between the two proteins [44-46]. Recently, some studies have shown that CAV-1, which is a protein responsible 
for regulating eNOS function, is closely linked to IGF-1 and IGFBP3, regulating endothelial cell proliferation, vascular development, and oxidative stress [47-52].

The SNPs of NOS3, rs1799983, TCF7L2 rs7903146, and IGFBP3 rs11977526, had genotypic frequencies similar to those observed in studies performed in African populations [40,53-55]. This study was part of a broader project, which aimed to diagnose nutritional and health conditions of the maternal and child population of quilombola communities in the state of Alagoas. Thus, it did not provide for the inclusion of men and elderly people. Therefore, the absence of men is a limitation of this research. Due to the differences in the occurrence of hypertension by gender, further studies including male participants should be conducted.

\section{Conclusion}

The TT genotype of the NOS3 rs1799983 gene and the AA genotype of the IGFBP3 rs11977526 gene are associated with a higher prevalence of arterial hypertension and are important risk factors for this condition, especially when associated with higher age groups, excess of body weight, diabetes, and dyslipidemia. Considering that the mechanism of action, responsible for higher blood pressure levels in women with the TT (NOS3) and AA (IGFBP3) genotypes, involves less metabolic production of nitric oxide and, consequently, an increase in oxidative stress, the results presented here suggest that these SNPs are directly related to blood pressure regulation.

Future molecular studies are needed to reveal the important roles of eNOS and IGFBP3 when they are related to hypertension. Association studies such as the one presented here are of great relevance for motivating research aimed to elucidate the molecular pathways involved in the etiology of hypertension and, consequently, in the development of new drugs related to these pathways.

\section{Abbreviations}

Asp: Aspartic acid

BMl: Body mass index

Cl: Confidence interval

CAV-1: Caveolin-1

DNA: Deoxyribonucleic acid

DM: Diabetes mellitus

DBP: diastolic blood pressure

EBIA: Brazilian Food Insecurity Scale 
eNOS: Nitric oxide synthase

FNS: Food and nutrition security

FNl: Food and nutrition insecurity

Glu: Glutamic acid

GWAS: Genome-Wide Association Studies

HbA1c: Glycated hemoglobin

HDL: High density lipoprotein

HWE: Hardy-Weinberg equilibrium

ICAM-1: Intercellular Adhesion Molecule 1

IGF-1: Insulin-like growth factor-1

IGFBP3: Insulin-like growth factor binding protein -3

IPAQ: Physical Activity Questionnaire

LDL: Low-density lipoprotein

NO: Nitric oxide

PAL: Physical activity level

PR: Prevalence ratio

SBP: systolic blood pressure

SNP: Single nucleotide polymorphism

WC: waist circumference

\section{Declarations}

\section{Ethics approval and consent to participate}

Ethics approval for this study was obtained from the Ethics Committee of Federal University of Alagoas, Brazil (No: 33527214.9.0000.5013). The study conformed to the principles of the Declaration of Helsinki. Written informed consent was obtained from all individual participants included in the study. 
Not applicable.

\section{Availability of data and material}

The datasets used and/or analyzed during the current study are available from the corresponding author on reasonable request.

\section{Competing interests}

The authors declare that they have no competing interests.

\section{Funding Statement}

This study was funded by the Brazilian National Council of Technological and Scientific Development CNPq (Grant $n^{\circ} 466718 / 2014-4$ ) and the Foundation for Research Support of the State of Alagoas Fapeal (Grant $\left.n^{\circ} 60030.000849 / 2016\right)$. The views expressed in the present article are those of the authors and not necessarily those of any funding agencies. The funders had no role in study design, data collection and analysis, decision to publish, or preparation of the manuscript.

\section{Authors' contributions}

A.B.L.N. participated in designing the study, analysis and interpretation of data and drafting the article. N.B.R.V, T.R.S, L.E.C.D, C.S.M and M.L.A. took part in the acquisition of data, data entry, analysis and interpretation and writing. H.S.F. took part in the project's conception and obtained the respective financial support, coordinating all implementation steps and realized the final review of the article. All authors approved the final version to be submitted.

\section{Acknowledgements}

For their support, National Council for Scientific and Technological Development (CNPq), National Council for the Improvement of Higher Education (CAPES), Foundation for Research Support of Alagoas state (FAPEAL), Federal University of Alagoas.

\section{References}

1. Geldsetzer P, Manne-Goehler J, Marcus M-E, Ebert C, Zhumadilov Z, Wesseh CS, Tsabedze L, Supiyev A, Sturua L, Bahendeka SK: The state of hypertension care in 44 low-income and middle-income countries: a cross-sectional study of nationally representative individual-level data from $1 \cdot 1$ million adults. The Lancet 2019, 394(10199):652-662.

2. Weber MA, Schiffrin EL, White WB, Mann S, Lindholm LH, Kenerson JG, Flack JM, Carter BL, Materson BJ, Ram CVS: Clinical practice guidelines for the management of hypertension in the community: a statement by the American Society of Hypertension and the International Society of Hypertension. The journal of clinical hypertension 2014, 16(1):14-26. 
3. Munroe PB, Barnes MR, Caulfield MJ: Advances in blood pressure genomics. Circulation research 2013, 112(10):1365-1379.

4. Doris PA: Hypertension genetics, single nucleotide polymorphisms, and the common disease: common variant hypothesis. Hypertension 2002, 39(2):323-331.

5. Franceschini N, Le TH: Genetics of hypertension: discoveries from the bench to human populations. American Journal of Physiology-Renal Physiology 2014, 306(1):F1-F11.

6. Briones AM, Touyz RM: Oxidative stress and hypertension: current concepts. Current hypertension reports 2010, 12(2):135-142.

7. Rizzoni D: Endothelial dysfunction in hypertension: fact or fantasy? Journal of hypertension 2002, 20(8):1479-1481.

8. Ferroni P, Basili S, Paoletti V, Davi G: Endothelial dysfunction and oxidative stress in arterial hypertension. Nutrition, metabolism and cardiovascular diseases 2006, 16(3):222-233.

9. Zhang L, Curhan GC, Forman JP: Plasma insulin-like growth factor-1 level and risk of incident hypertension in non-diabetic women. Journal of hypertension 2011, 29(2):229.

10. Sowers JR: Insulin and insulin-like growth factor in normal and pathological cardiovascular physiology. Hypertension 1997, 29(3):691-699.

11. Schutte AE, Volpe M, Tocci G, Conti E: Revisiting the relationship between blood pressure and insulinlike growth factor-1. Hypertension 2014, 63(5):1070-1077.

12. Conen D, Ridker PM, Mora S, Buring JE, Glynn RJ: Blood pressure and risk of developing type 2 diabetes mellitus: the Women's Health Study. European heart journal 2007, 28(23):2937-2943.

13. Ferrannini E, Cushman WC: Diabetes and hypertension: the bad companions. The Lancet 2012, 380(9841):601-610.

14. Yan Y, Klein R, Heiss G, Girman CJ, Lange EM, Klein BE, Rose KM, Boerwinkle E, Pankow JS, Brancati FL: The transcription factor 7-like 2 (TCF7L2) polymorphism may be associated with focal arteriolar narrowing in Caucasians with hypertension or without diabetes: the ARIC Study. BMC endocrine disorders 2010, 10(1):9.

15. Senthil D, Raveendran M, Shen YH, Utama B, Dudley D, Wang J, Wang XL: Genotypedependent expression of endothelial nitric oxide synthase (eNOS) and its regulatory proteins in cultured endothelial cells. DNA and cell biology 2005, 24(4):218-224.

16. Padilha BM, da Silva Diniz A, da Silva Ferreira H, Tomiya MTO, Cabral PC: Anthropometric predictors of hypertension in afro-descendant women. Scientia Medica 2017, 27(3):27527.

17. Chobanian AV, Bakris GL, Black HR, Cushman WC, Green LA, Izzo Jr JL, Jones DW, Materson BJ, Oparil S, Wright Jr JT: The seventh report of the joint national committee on prevention, detection, evaluation, and treatment of high blood pressure: the JNC 7 report. Jama 2003, 289(19):2560-2571.

18. Neto ABL, Farias MC, Vasconcelos NB, Xavier JA, Assunção ML, Ferreira HS: Prevalence of endothelial nitric oxide synthase (ENOS) gene G894T polymorphism and its association with 
hypertension: a population-based study with Brazilian women. Archives of medical sciences Atherosclerotic diseases 2019, 4:e63-e73.

19. Yoshimura M, Yasue H, Nakayama M, Shimasaki Y, Sumida H, Sugiyama S, Kugiyama K, Ogawa H, Ogawa Y, Saito Y: A missense Glu298Asp variant in the endothelial nitric oxide synthase gene is associated with coronary spasm in the Japanese. Human genetics 1998, 103(1):65-69.

20. Kaplan RC, Petersen A-K, Chen M-H, Teumer A, Glazer NL, Döring A, Lam CS, Friedrich N, Newman A, Müller M: A genome-wide association study identifies novel loci associated with circulating IGF-I and IGFBP-3. Human molecular genetics 2011, 20(6):1241-1251.

21. Sousa AGP, Marquezine GF, Lemos PA, Martinez E, Lopes N, Hueb WA, Krieger JE, Pereira AC: TCF7L2 polymorphism rs7903146 is associated with coronary artery disease severity and mortality. PLOS One 2009, 4(11).

22. Bush WS, Moore JH: Genome-wide association studies. PLoS computational biology 2012, 8(12).

23. Hirschhorn JN, Daly MJ: Genome-wide association studies for common diseases and complex traits. Nature reviews genetics 2005, 6(2):95-108.

24. Kepple AW, Segall-Corrêa AM: Conceituando e medindo segurança alimentar e nutricional. Ciência \& Saúde Coletiva 2011, 16:187-199.

25. Segall-Corrêa AM, Marin-León L, Melgar-Quiñonez H, Pérez-Escamilla R: Refinement of the Brazilian household food insecurity measurement scale: Recommendation for a 14-item EBIA. Revista de Nutrição 2014, 27(2):241-251.

26. Lee PH, Macfarlane DJ, Lam TH, Stewart SM: Validity of the international physical activity questionnaire short form (IPAQ-SF): A systematic review. International Journal of Behavioral Nutrition and Physical Activity 2011, 8(1):115.

27. Caminha TC, Ferreira HS, Costa NS, Nakano RP, Carvalho RES, Xavier Jr AF, Assunção ML: Waist-toheight ratio is the best anthropometric predictor of hypertension: A population-based study with women from a state of northeast of Brazil. Medicine 2017, 96(2).

28. Martin SS, Blaha MJ, Elshazly MB, Toth PP, Kwiterovich PO, Blumenthal RS, Jones SR: Comparison of a novel method vs the Friedewald equation for estimating low-density lipoprotein cholesterol levels from the standard lipid profile. Jama 2013, 310(19):2061-2068.

29. Scartezini M, Ferreira CES, Izar MCO, Bertoluci M, Vencio S, Campana GA, Sumita NM, Barcelos LF, Faludi AA, Santos RD et al: Positioning about the Flexibility of Fasting for Lipid Profiling. Arquivos Brasileiros de Cardiologia 2017, 108:195-197.

30. Wigginton JE, Cutler DJ, Abecasis GR: A note on exact tests of Hardy-Weinberg equilibrium. The American Journal of Human Genetics 2005, 76(5):887-893.

31. Solé X, Guinó E, Valls J, Iniesta R, Moreno V: SNPStats: a web tool for the analysis of association studies. Bioinformatics 2006, 22(15):1928-1929.

32. Barnard SA, Smith W, Mels CM, Botha S, Schutte AE: Bioavailable IGF-1 is beneficially associated with biomarkers of endothelial function in young healthy adults: The African-PREDICT study. Growth Hormone \& IGF Research 2018, 41:28-33. 
33. Balaram SK, Agrawal DK, Allen RT, Kuszynski CA, Edwards JD: Cell adhesion molecules and insulinlike growth factor-1 in vascular disease. Journal of vascular surgery 1997, 25(5):866-876.

34. Al-Zahrani A, Sandhu MS, Luben RN, Thompson D, Baynes C, Pooley KA, Luccarini C, Munday H, Perkins B, Smith P: IGF1 and IGFBP3 tagging polymorphisms are associated with circulating levels of IGF1, IGFBP3 and risk of breast cancer. Human molecular genetics 2006, 15(1):1-10.

35. Cheng I, DeLellis Henderson K, Haiman CA, Kolonel LN, Henderson BE, Freedman ML, Le Marchand Lc: Genetic determinants of circulating insulin-like growth factor (IGF)-I, IGF binding protein (BP)-1, and IGFBP-3 levels in a multiethnic population. The Journal of Clinical Endocrinology \& Metabolism 2007, 92(9):3660-3666.

36. Sakar MN, Atay AE, Demir S, Bakir VL, Demir B, Balsak D, Akay E, Ulusoy Al, Verit FF: Association of endothelial nitric oxide synthase gene G894T polymorphism and serum nitric oxide levels in patients with preeclampsia and gestational hypertension. The Journal of Maternal-Fetal \& Neonatal Medicine 2015, 28(16):1907-1911.

37. Ma Q, Huang K, Guo H, Yang W, Luo W, Qiu J, Yang L: Endothelial nitric oxide synthase gene G894T polymorphism and risk assessment for pregnancy-induced hypertension: evidence from 11700 subjects. Hypertension Research 2016, 39(12):899-906.

38. ALrefai AA, Habib MSE-d, Yaseen RI, Gabr MK, Habeeb RM: Association of endothelial nitric oxide synthase (eNOS) gene G894T polymorphism with hypertension risk and complications. Molecular and cellular biochemistry 2016, 421(1-2):103-110.

39. Goni L, Cuervo M, Milagro FI, Martínez JA: Influence of fat intake and BMI on the association of rs1799983 NOS3 polymorphism with blood pressure levels in an Iberian population. European journal of nutrition 2017, 56(4):1589-1596.

40. Kayima J, Liang J, Natanzon Y, Nankabirwa J, Ssinabulya I, Nakibuuka J, Katamba A, Mayanja-Kizza $\mathrm{H}$, Miron A, Li C: Association of genetic variation with blood pressure traits among East Africans. Clinical genetics 2017, 92(5):487-494.

41. Liang J, Le TH, Edwards DRV, Tayo BO, Gaulton KJ, Smith JA, Lu Y, Jensen RA, Chen G, Yanek LR: Single-trait and multi-trait genome-wide association analyses identify novel loci for blood pressure in African-ancestry populations. PLoS genetics 2017, 13(5):e1006728.

42. Philip I, Plantefeve G, Vuillaumier-Barrot S, Vicaut E, LeMarie C, Henrion D, Poirier O, Levy BI, Desmonts JM, Durand Gv: G894T polymorphism in the endothelial nitric oxide synthase gene is associated with an enhanced vascular responsiveness to phenylephrine. Circulation 1999, 99(24):3096-3098.

43. Fang Z, Yang S, Zhu L, Li Y, Chen Y, Jin Y, Zhao X, Zhao H, Chen X, Zhao Y: Association study of IGFBP1 and IGFBP3 polymorphisms with hypertension and cardio-cerebral vascular diseases in a Chinese Han population. Oncotarget 2017, 8(44):77836.

44. Matsumoto M, Hatakeyama S, Oyamada K, Oda Y, Nishimura T, Nakayama KI: Large-scale analysis of the human ubiquitin-related proteome. Proteomics 2005, 5(16):4145-4151. 
45. Singh G, Kucukural A, Cenik C, Leszyk JD, Shaffer SA, Weng Z, Moore MJ: The cellular EJC interactome reveals higher-order mRNP structure and an EJC-SR protein nexus. Cel/ 2012, 151(4):750-764.

46. Petschnigg J, Groisman B, Kotlyar M, Taipale M, Zheng Y, Kurat CF, Sayad A, Sierra JR, Usaj MM, Snider J: The mammalian-membrane two-hybrid assay (MaMTH) for probing membrane-protein interactions in human cells. Nature methods 2014, 11(5):585.

47. Bahr TM, Hughes GJ, Armstrong M, Reisdorph R, Coldren CD, Edwards MG, Schnell C, Kedl R, LaFlamme DJ, Reisdorph N: Peripheral blood mononuclear cell gene expression in chronic obstructive pulmonary disease. American journal of respiratory cell and molecular biology 2013, 49(2):316-323.

48. Shyu H-Y, Chen M-H, Hsieh Y-H, Shieh J-C, Yen L-R, Wang H-W, Cheng C-W: Association of eNOS and Cav-1 gene polymorphisms with susceptibility risk of large artery atherosclerotic stroke. PloS one 2017, 12(3).

49. Jarajapu YP, Cai J, Yan Y, Calzi SL, Kielczewski JL, Hu P, Shaw LC, Firth SM, Chan-Ling T, Boulton ME: Protection of blood retinal barrier and systemic vasculature by insulin-like growth factor binding protein-3. PLoS One 2012, 7(7).

50. Beyfuss K, Hood DA: A systematic review of p53 regulation of oxidative stress in skeletal muscle. Redox report 2018, 23(1):100-117.

51. Aharinejad S, Salama M, Rödler S, Ehrlich M, Zuckermann A, Laufer G: Low serum IGF-1 is a risk factor for cardiac allograft vasculopathy in cardiac transplant recipients. Transplantation 2012, 93(3):309-313.

52. Yamaguchi Y, Yasuoka H, Stolz DB, Feghali-Bostwick CA: Decreased caveolin-1 levels contribute to fibrosis and deposition of extracellular IGFBP-5. Journal of cellular and molecular medicine 2011, 15(4):957-969.

53. Li R, Lyn D, Lapu-Bula R, Oduwole A, Igho-Pemu P, Lankford B, Morgan J, Nkemdechi S, Liu G, Pack C: Relation of endothelial nitric oxide synthase gene to plasma nitric oxide level, endothelial function, and blood pressure in African Americans. American journal of hypertension 2004, 17(7):560-567.

54. Gad MZ, Abdel Rahman MF, Hashad IM, Abdel-Maksoud SM, Farag NM, Abou-Aisha K: Endothelial nitric oxide synthase (G894T) gene polymorphism in a random sample of the Egyptian population: comparison with myocardial infarction patients. Genetic testing and molecular biomarkers 2012, 16(7):695-700.

55. Danquah I, Othmer T, Frank LK, Bedu-Addo G, Schulze MB, Mockenhaupt FP: The TCF7L2 rs7903146 (T) allele is associated with type 2 diabetes in urban Ghana: a hospital-based case-control study. BMC medical genetics 2013, 14(1):96.

\section{Figures}




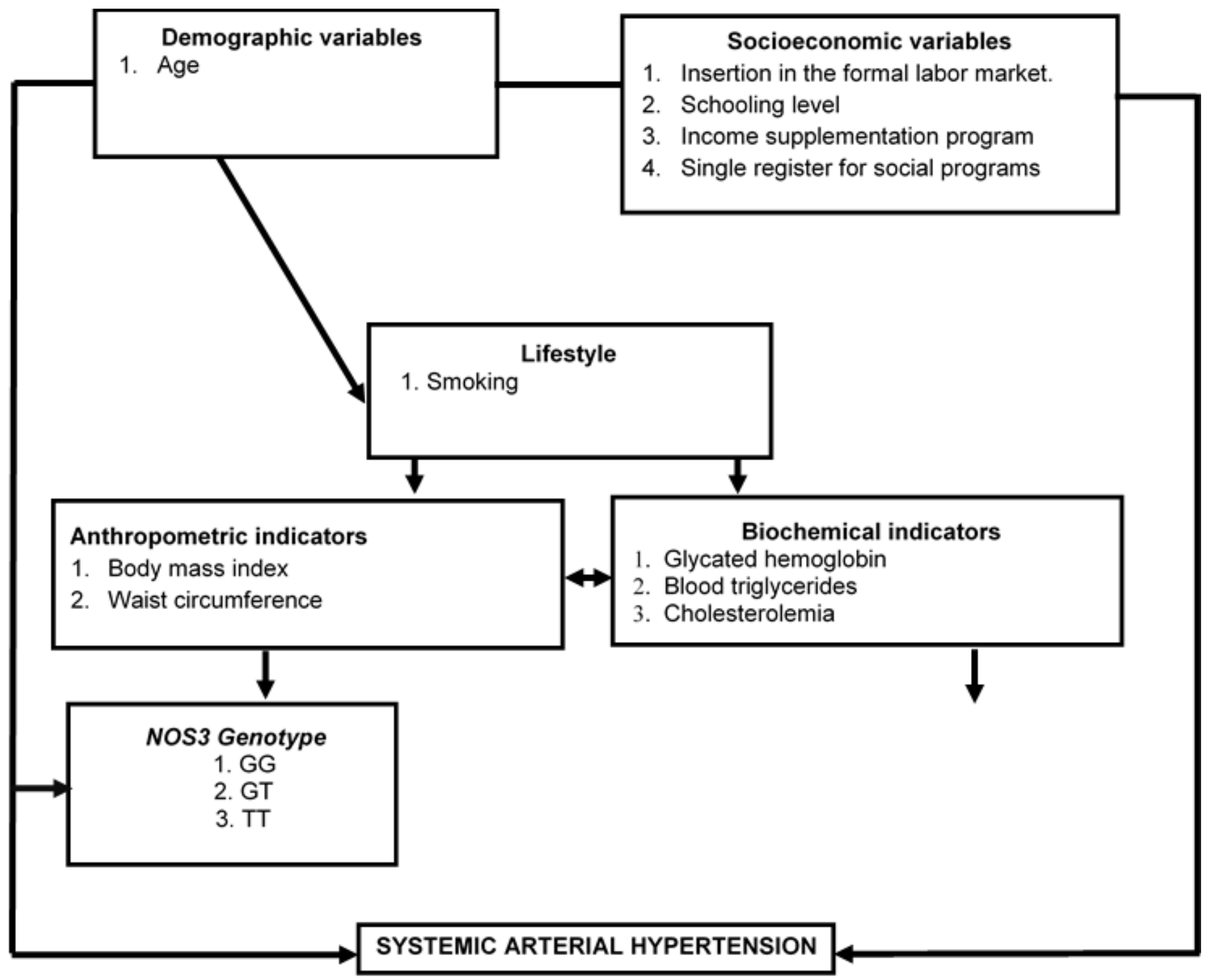

Figure 1

Hierarchical conceptual model explaining systemic arterial hypertension (Adapted from Neto et al., 2019). 